№.........

ИНСТИТУТ ПО МАТЕМАТИКА С ИЗЧИСЛИТЕЛЕН ЦЕНТЬР INSTITUTE OF MATHEMATICS WITH COMPUTER CENTER

\title{
Kuratowski convergence of the efficient sets in the parametric linear vector semi-infinite optimization
}

БЪЛГАРСКА АКАДЕМИЯ HA НАУКИTE

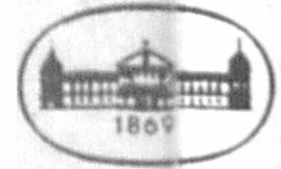

BULGARIAN ACADENY OF SCIENCES
Preprint

November 1993

No 15 


\title{
Kuratowski convergence of the efficient sets in the parametric linear vector semi-infinite optimization*
}

\author{
Maxim Ivanov Todorov
}

Bulgarian Academy of Sciences, Institute of Mathematics 29 Ph. Macedonsky Str., r. 401, 4002 Plovdiv, BULGARIA

\begin{abstract}
In the space of whole linear semi-infinite optimization problems we consider the mappings putting into correspondance to each problem the set of efficient and weakly efficient points, respectively. We endow the image space wit Kuratowski convergence and by means of the lower and upper continuity of these mappings we prove generic well-posedness of the vector optimization problems. The connection between the continuity and some properties of the efficient sets is also discussed.
\end{abstract}

KEY WORDS Linear vector semi-infinite optimization, lower and upper semi- continuity, Kuratowski convergence, well-posedness.

*This work was partially supported by grant MM-21 of the Bulgarian Ministry of Education and Science 


\section{Introduction}

Many authors like Tanino [1], Bednarczuk [2] and s.o. have treated the question about the upper and lower semi-continuity of the efficient maps in the vector optimization. In two previous papers we have investigated the continuity properties of the efficient sets in the linear vector semi-infinite optimization. Comparing the results in these articles we can see that there exists something like duality between the continuity properties of the weakly efficient and efficient sets. This duality with respect to the upper and lower semi-continuity helps us to create interesting and internal characterizations of both sets. For example some topological properties like closedness of the set of efficient points, coincidness of the sets of weakly efficient and efficient points and well-posedness in the vector optimization.

Now let us describe what does it mean linear vector semi-infinite optimization problem and since we shall write about continuity we have to define the parameter space of such kind of problems.

Let $\mathrm{T}$ be a compact Hausdorff space and $R^{N}$ be the usual $\mathrm{N}$-dimensional Eucledean space. Let $B: T \longrightarrow R^{N}$ and $b: T \longrightarrow R$ be continuous mappings and $\left(p_{1}, p_{2}, \ldots, p_{l}\right)$ be elements in $R^{N}$.

We define $P: R^{N} \longrightarrow R^{l}$ by setting: For every $x \in R^{N}$

$$
P(x)=\left(<p_{1}, x>,<p_{2}, x>, \ldots,<p_{l}, x>\right)
$$

For each triplet

$$
\sigma=(B, b, P) \in\left\{(C(T))^{N} \times C(T) \times R^{N \times l}\right\}=\Theta
$$

as in [3] we consider the closed set $Z(\sigma) \subset R^{N}$ described by side-conditions:

$$
Z(\sigma)=\left\{x \in R^{N}: \text { for every } t \in T\right\}
$$

and the following linear vector optimization problems.

$L V M(\sigma)$ : Determine an efficient poin subject to the side- conditions.

$L V W(\sigma)$ : Determine a weakly efficient poin subject to the side-conditions.

Here we have used the following:

First, In the finite dimensional space $R^{l}$ we consider the partial ordering generated by the cone $R_{+}^{i}$. This means that for any $z^{1}, z^{2} \in R^{i}, z^{1} \leq z^{2}$ iff this inequality holds for the coresponding coordinates of $z^{1}$ and $z^{2}$. And second the usual definitions: 
Definition 1.1 A point $x_{0} \in Z(\sigma)$ is called efficient iff for each $x \in Z(\sigma)$ such that $P(x) \leq P\left(x_{0}\right)$ holds $P(x)=P\left(x_{0}\right)$.

Definition $1.2 A$ point $x_{0} \in Z(\sigma)$ is called weakly efficient iff for each $x \in$ $Z(\sigma)$ such that $P(x) \leq P\left(x_{0}\right)$ holds that there exists $j \in \overline{1, l}$ for which $<p_{j}, x>=<p_{j}, x_{0}>$.

Obviously each efficient point is a weakly efficient point but the converse statement is not true in general.

In the space $\Theta$ we consider the norm:

$$
\begin{gathered}
\|x\|_{R^{N}}=\max _{1 \leq i \leq N}\left|x_{i}\right|, \quad\|P\|_{R^{N \times l}}=\max _{1 \leq i \leq l}\left\|P_{i}\right\|_{R^{N}} \\
\|b\|_{\infty}=\max _{t \in T}|b(t)|, \quad\|B\|_{\infty}=\max _{t \in T}\|B(t)\|_{R^{N}} \\
\text { and for every } \sigma=(B, b, P) \in \Theta \\
\|\sigma\|=\|B\|_{\infty}+\|b\|_{\infty}+\|P\|_{R^{N \times l}} .
\end{gathered}
$$

This norm turns $\Theta$ into a Banach space and generates in $\Theta$ the nature cartesian product topology.

For each $\sigma \in \Theta$ we put:

$$
\begin{gathered}
F(\sigma)=\{x \in Z(\sigma): \quad \mathrm{x} \text { is an efficient point }\} \\
F W(\sigma)=\{x \in Z(\sigma): \quad \mathrm{x} \text { is a weakly efficient point }\}
\end{gathered}
$$

The multivalued mapping $F: \Theta \longrightarrow R^{N}$ puts into correspondance to every $\sigma \in \Theta$ the set of efficient points and $F W: \Theta \longrightarrow R^{N}$ the set of weakly efficient points respectilely.

Before to go further we shall set the definitions of the continuity of the set-valued maps.

Definition 1.3 We shall say that the multivalued map $F: X \longrightarrow Y$ between the topological spaces $X$ and $Y$ is upper (lower) semi-continuous at the point $x_{0} \in X$ iff for every open nghb $W \supset F\left(x_{0}\right)\left(W \cap F\left(x_{0}\right) \neq \emptyset\right)$ there exists an open nghb $V \ni x_{0}$ such that for each $x \in V$ holds $F(x) \subset W(W \cap F(x) \neq \emptyset)$.

There are several definitions of well-posedness in the vector optimization due to Penno and Bednarczuk [4], Tanino and Sawaragi [5], Luccetti [6] and s.o. In this article we shall consider this given by Tanino and Sawaragi because it is more suitable for our purposes. 
Definition 1.4 The problem $L V M(\sigma)(L V W(\sigma))$ is well-posed iff the mapping $F(F W)$ is continuous at the point $\sigma \in \Theta$, i.e. $F(F W)$ is lower and upper semi-continuous at $\sigma \in \Theta$.

As it is seen from the definition the well-posedness depends strongly on the topologies chosen in the parameter and image spaces. To avoid this obstacle it would be nice to search into two different directions.

First, which was a metter of the previous papers [7,8], is to look for good properties giving an information about the continuity without having in mind the topologies in both spaces. We shall mention here so called "domination properties" [2] and this one given in [7]:

Definition 1.5 We shall say that the point $\sigma \in \Theta$ is "nice" if the sets of efficient and weakly efficient points coincide.

It turns out that the "nice" property is closely related to the well-posedness of the problem, nevertheless there are not topological notions in it. This property guarantees also the closedness of the set $F(\sigma)$ which is not true in general and it could be interpreted as the uniqueness in the definition of well-posedness in the scalar optimization.

Second direction, which is the main aim of the paper is to find suitable topologies, especialy in the image space, giving us the possibility to prove easily well-posedness not only of the restricted maps. On the base of linear vector semi-infinite optimization we shall show an example how to apply the new ideas of the topologies in the space of closed subsets of a Banach space. It is obvious that we can not prove generic well-posedness if we use the well known Hausdorff topology, so we must apply other ones like Mosco, or bounded Hasdorff topology [9] and s.o. In the last part of the article we prove the Kuratowski convergence, which is a base of these new concepts, of the efficient sets in the parametric linear vector semi-infinite optimization.

In the next section we shall present a short review of the known results in the area and in the second part making use of them we shall state the proofs of the basic aasertions in our article.

\section{Properties of the efficient sets}

In this section we shall present some continuity results and by means of them we show that the above defined "nice" property is essential, i.e. it is fulfilled 
in a dense subset of the set of solvable problems.

We need several definitions and statements

$$
\begin{gathered}
L_{Z}=\{\sigma \in \Theta: Z(\sigma) \neq \emptyset\} \\
L_{M}=\{\sigma \in \Theta: L V M(\sigma) \text { has a solution }\} \\
L_{W}=\{\sigma \in \Theta: L V W(\sigma) \text { has a solution }\}
\end{gathered}
$$

Let $\mathrm{A}$ be a subset of a topological space $\mathrm{X}$. int A denotes the set of all interior points of A.

Let us remind that the sets $L_{M}$ and $L_{W}$ do not coincide and also a theorem given in [10].

Theorem 2.1 Let compact $T$ contain at least $N$ points. Then

$$
\emptyset \neq \operatorname{int} L_{M} \subset L_{M} \subset L_{W} \subset \overline{\operatorname{int} L_{M}} .
$$

Definition 2.2 We shall say that the Slater condition is fulfilled for $\sigma \in \Theta$ iff there exists a point $x \in R^{N}$ such that $\quad\langle B(t), x\rangle<b(t) \quad$ for every $t \in T$.

Remark: It is easy to see that $\sigma \in$ int $L_{Z}$ iff the Slater condition is fulfilled.

For every $i=1,2, \ldots$ we define

$$
\begin{aligned}
& B_{i} \cdot \text { closed ball in } R^{N} \text { with a radius } \mathrm{i} \\
& F W_{i}: \Theta \longrightarrow B_{i}, \quad \text { where } \\
& F W_{i}(\sigma)=\left\{F(\sigma) \cap B_{i}\right\} \\
& A W_{i}=\left\{\sigma \in \operatorname{int} L_{Z}: F W_{i}(\sigma) \neq \emptyset\right\} .
\end{aligned}
$$

In [7], using a direct aproach, we have proved for the restricted maps $F W_{i}$ the following:

Theorem 2.3 For every $i=1,2, \ldots$ the multivalued mapping $F W_{i}$ is upper semi-continuous at each point $\sigma \in A W_{i}$.

According to the famous theorem of Fort [11]

Theorem 2.4 Let $X$ be topological space and $Y$ a metric space. Let $F$ : $X \longrightarrow 2^{Y}$ be and upper (lower) semi-continuous and compact valued mapping. Then the set of points $x \in X$ where $F$ is not lower (upper) semicontinuous is of the first Baire category in $X$. 
we get a theorem which gives us well-posedness but only of the restricted over the bals problems $L V W(\sigma)$ :

Theorem 2.5 For every $i=1,2, \ldots$ there exists a dense and $G_{\delta}$ subset $M W_{i}$ of $A W_{i}$ sush that the multivalued mapping $F W_{i}$ is upper and lower semi-continuous at each point $\sigma \in M W_{i}$.

In order to present the symmetric statement for the map $\mathrm{F}$ we have to put some restrictions.

1. Let from now on $l \geq N$.

2. We shall consider only the set

$\Theta L=\left\{\sigma \in \Theta: \operatorname{rank} P=N\right.$ and $\sigma \in \operatorname{int} L_{Z}$ and $\left.\operatorname{rank}[B(t), t \in T]=N\right\}$. In [12] we have proved that:

Theorem 2.6 The set $\Theta L$ is an open and dense subset of the space $\Theta$.

For every $i=1,2, \ldots$ we define

$$
\begin{aligned}
& F_{i}: \Theta L \longrightarrow B_{i}, \quad \text { where } \\
& F_{i}(\sigma)=\left\{x \in Z(\sigma) \cap B_{i}: \quad x \text { is an efficient point }\right\} \\
& Z_{i}(\sigma)=\left\{x \in B_{i}: \quad \text { for every } t \in T\right\} .
\end{aligned}
$$

In [8], this time by means of a sufficient condition given in [1] we have got

Theorem 2.7 For every $i=1,2, \ldots$ and $\sigma \in \Theta L$ such that int $Z_{i}(\sigma) \neq \emptyset$ the mapping $F_{i}$ is lower semi- continuous at the point $\sigma$.

Theorems 2.3 and 2.7 clarify what we mean with the duality. When we speak about weak efficiency we have upper semi-continuity and with efficiency lower semi-continuity, respectively. We should mention here that these results are valid only for the rstricted maps and the duality can not be understood in the terms of theorem 2.5. The reason that we are not able to obtain a similar statement to this one in theorem 2.5, for the map F is that in the theorem of Fort one needs of the compact images. Unfortunately, the set $F(\sigma)$ is not closed in general. Because of that we shall present a sufficient condition which guarantees the closedness. This property is introdused by Wegmann [13]. 
Definition 2.8 The set $Z$ has a property $\left(P^{*}\right)$ iff for every $x, z \in Z$ there exists $\epsilon>0$ such that for each $v \in O_{\epsilon}(x) \cap Z$ there exists $\alpha>0$ such that

$$
v+\alpha(z-x) \in Z \text {. }
$$

Remark: The intersection of finitely many $\left(P^{*}\right)$-sets is also $\left(P^{*}\right)$-set. With the norm introduced at the beginning the unit ball in $R^{N \times l}$ has a property $\left(P^{*}\right)$.

Lemma 2.9 If the feasible set $Z(\sigma)$ has the property $\left(P^{*}\right)$ then the set of efficient points $F(\sigma)$ is closed for each $P \in R^{N \times l}$.

Proof: We shall write $F(P)$ and $\mathrm{Z}$ insted of $F(\sigma)$ and $Z(\sigma)$ because we vary only P.

If $F(P)=\emptyset$ there is nothing to prove. Let $F(P) \neq \emptyset$ and let us assume that $\mathrm{F}(\mathrm{P})$ is not closed, i.e. there exists a sequence $x_{n} \frac{\|\| \|}{n \rightarrow \infty}>x_{0}$ such that $x_{n} \in F(P), \quad n=1,2, \ldots$ and $x_{0} \notin F(P)$. This means that we can find $y \in Z$ and $j \in \widehat{1, l}$ such that

$$
P(y) \leq P\left(x_{0}\right) \quad \text { and } \quad\left\langle p_{j}, y><<p_{j}, x_{0}>.\right.
$$

The feasible set $\mathrm{Z}$ is a $\left(P^{*}\right)$-set therefore there exists $\epsilon>0$ such that for every $v \in O_{\epsilon}\left(x_{0}\right) \cap Z$ there exists $\alpha>0$ with $v+\alpha\left(y-x_{0}\right) \in Z . x_{n} \frac{\|H\|}{n-\infty}>x_{0}$ whereby we can find $\mathrm{m}$ cuch that for each $n \geq m$ holds $x_{n} \in O_{\epsilon}\left(x_{0}\right) \cap Z$ We fix such $\mathrm{n}$ and take $\alpha_{n}>0$ such that $x_{n}+\alpha_{n}\left(y-x_{0}\right) \in Z$.

Now we have

$$
\begin{gathered}
P\left[x_{n}+\alpha_{n}\left(y-x_{0}\right)\right]=P\left(x_{n}\right)+\alpha_{n} P\left(y-x_{0}\right) \leq P\left(x_{0}\right), \quad \text { and } \\
\left.<p_{j}, x_{n}+\alpha_{n}\left(y-x_{0}\right)>=<p_{j}, x_{n}>+\alpha_{n}<p_{j}, y-x_{0}><<p_{j}, x_{n}\right\rangle .
\end{gathered}
$$

But this is a contradiction with the fact that $x_{n} \in F(P)$. The proof is completedo

The $\left(P^{*}\right)$-propery is not too strong restriction, f.e. the convex sets in $R^{2}$, the polychedrons in $R^{n}$, etc. are $\left(P^{*}\right)$-sets. For the wide class compacta T, the problems with $\left(P^{*}\right)$-feasible set form a dense subset of the set $L_{Z}$. The folowing theorem from [8] is true.

Theorem 2.10 Let $T$ be a homeomorphic space to the unit cube in some $R^{m}$, or finite union of such kind of spaces. Then set of problems $\sigma \in L_{Z}$ such that the feasible set $Z(\sigma)$ is a polychedron is a dense subset of the set $L_{Z}$. 
We should say that the spaces homeomorphic to the unit cube in $R^{m}$ are a rather large class. Each convex compact in a Eucledean space belongs to this class. One can find many haracterizations of such homeomorphizms with different $\mathrm{m}$ in the topological literature.

Now according to the fact that when the feasible set has $\left(P^{*}\right)$-property the set of efficient point is closed, we are ready to present the assertion similar to a theorem 2.5 , but only with fixed $\left(P^{*}\right)$-feasible set and $\mathrm{P}$ variable.

Let us define the sets:

$$
\begin{gathered}
A=\left\{P \in R^{N \times l}: F(P) \neq \emptyset\right\}, \quad \text { and } \\
A_{i}=\left\{P \in R^{N \times l}: F(P) \cap \text { int } B_{i} \neq \emptyset\right\}, \quad i=1,2, \ldots . \\
\text { Obviously } \quad A=\bigcup_{i=1}^{\infty} A_{i} .
\end{gathered}
$$

Using the same sheme with the theorem of Fort in [8] the following theorem has been proved

Theorem 2.11 For every $i=1,2, \ldots$ such that $A_{i} \neq \emptyset$ there exists a dense and $G_{\delta}$ subset $M_{i}$ of $A_{i}$ such that at each $P \in M_{i}$ the mapping $F_{i}$ is both upper and lower semi-continuous.

From this very alike to a well-posedness statement, also in [8] we get

Theorem 2.12 Let the feasible set $Z$ be fixed and have the property $\left(P^{*}\right)$ then the set of problems which are not "nice" is of the first Baire category.

We have the "nice" property as a cosequence of the upper semi-continuity of the maps $F_{i}, i=1,2, \ldots$, but we must remind that to obtain upper, first we need of the lower semi-continuity. So we see that the "nice" property is very closely related to the well-posedness.

Now we shall prove the density of the "nice" property when we vary the whole parameter $\sigma=(B, b, P)$.

Theorem 2.13 Let the compact $T$ be homeomorphic to the unit cube in a Eucledean space. Then $\left\{\sigma \in L_{W}: \sigma\right.$ is "nice" $\}$ is a dense subset of the set A. 
Proof: Let us take $\sigma_{0}=\left(B_{0}, b_{0}, P_{0}\right) \in L_{W}$ and fix $\epsilon>0$. Having in mind theorems 2.1 and 2.10 , we find a point $\sigma_{1}=\left(B_{\epsilon}, b_{\epsilon}, P_{0}\right) \in L_{M}$ such that $Z\left(\sigma_{1}\right)$ is a $\left(P^{*}\right)$-set and $\left\|\sigma_{1}-\sigma_{0}\right\|<\epsilon / 2$. According to a theorem 2.12 we choose a point $\sigma_{\epsilon}=\left(B_{\epsilon}, b_{\epsilon}, P_{\epsilon}\right) \in L_{M}$ such that the problem $\sigma_{\epsilon}$ is "nice" and $\left\|\sigma_{1}-\sigma_{\epsilon}\right\|<\epsilon / 2$. Then

$$
\left\|\sigma_{0}-\sigma_{\epsilon}\right\| \leq\left\|\sigma_{0}-\sigma_{1}\right\|+\left\|\sigma_{1}-\sigma_{\epsilon}\right\|<\epsilon / 2+\epsilon / 2=\epsilon .
$$

The theorem is provedo

\section{Kuratowski continuity of the efficient maps}

In the space $2^{R^{n}}$, according to [14], we make the following notations and definitions. Let us take a sequence $\left\{A_{n}\right\}_{n \geq 1} \subset 2^{R^{n}}$ :

$$
\begin{gathered}
\operatorname{Li}_{n \rightarrow \infty} A_{n}=\left\{x: \exists\left\{x_{i}\right\}_{i \geq 1} ; x_{i} \in A_{i}, i=1,2, \ldots \text { and } x_{i} \rightarrow_{i \rightarrow \infty} x\right\} \\
L s_{n \rightarrow \infty} A_{n}=\left\{x: \exists k_{1}<k_{2}<\ldots ; x_{k_{i}} \in A_{k_{i}}, i=1,2, \ldots \text { and } x_{k_{i}} \rightarrow_{i \rightarrow \infty} x\right\}
\end{gathered}
$$

The sets $L i_{n \rightarrow \infty} A_{n}$ and $L s_{n \rightarrow \infty} A_{n}$ are closed and also $L i_{n \rightarrow \infty} A_{n} \subset L s_{n \rightarrow \infty} A_{n}$.

Definition 3.1 We say that the set $A$ is a Kuratowski limit of the sequence $\left\{A_{n}\right\}_{n \geq 1}$ iff $A=L i_{n \rightarrow \infty} A_{n}=L s_{n \rightarrow \infty} A_{n}$.

Another equivalent definition reads as follows

Definition 3.2 We say that the set $A$ is a Kuratowski limit of the sequence $\left\{A_{n}\right\}_{n \geq 1}$ iff

1. $\forall x \in A \exists\left\{x_{i}\right\}_{i \geq 1}, x_{i} \in A_{i}, i=1,2, \ldots$ such that $x_{i} \longrightarrow i \rightarrow \infty x$.

2. $\forall k_{1}<k_{2}<\ldots, x_{k_{i}} \in A_{k_{i}}, i=1,2, \ldots$ and $x_{k_{i}} \rightarrow i \rightarrow \infty x$ holds $x \in A$.

The next theotem from [3] givess the connection between the lower semicontinuity of the multivalued mappings and the lower Kuratowski limits.

Theorem 3.3 The multivalued mapping $F: X \rightarrow Y$ is lower semicontinuous at the point $x \in X$ iff for every sequence $\left\{x_{i}\right\}_{i \geq 1}$ such that $x_{i} \longrightarrow i \rightarrow \infty$ holds

$$
F(x) \subset L i_{i \rightarrow \infty} F\left(x_{i}\right) \text {. }
$$


Now we shall show generic well-posedness not only of the restricted maps. First we are going to do this for the mapping F. Let us endow the space $2^{R^{N \times i}}$ with the Kuratowski convergence. Since the set of efficient points is not always closed we need of a fixed $\left(P^{*}\right)$-feasible set and of the defined in the previous part sets $A, A_{i}$, and $M_{i}, i=1,2, \ldots$.

We shall construct new dense and $G_{\delta}$ subsets $N_{i}$ of $A_{i}, i=1,2, \ldots$. From [8] we have that $A_{i}$ are open sets and $A_{i} \subset A_{i+1}, i=1,2, \ldots$.

$$
\begin{aligned}
& N_{1}=M_{1}, \\
& N_{i}=\left\{\cap_{j=1}^{i-1}\left[M_{j} \cup\left(A_{i} \backslash \bar{A}_{j}\right)\right] \cap_{j=i}^{\infty} M_{j}\right\}, i=2,3, \ldots .
\end{aligned}
$$

Proposition 3.4 For all $i=1,2, \ldots$ the set $N_{i}$ is a dense and $G_{6}$ subset of the set $A_{i}$.

Proof: It is enough to prove only that for some i $M_{i} \cup\left(A \backslash \bar{A}_{i}\right)$ is a dense and $G_{\delta}$ subset of A.

The density is obvious. Sinse $M_{i}$ is a dense and $G_{\delta}$ subset of $A_{i}$ we can present it as $M_{i}=\cap_{j=i}^{\infty} M_{j}^{i}$, where $M_{j}^{i}$ are open and dense subsets of $A_{i}$, $j=1,2, \ldots$. Then

$$
M_{i} \cup\left(A \backslash \bar{A}_{i}\right)=\left(\cap_{j=i}^{\infty} M_{j}^{i}\right) \cup\left(A \backslash \bar{A}_{i}\right)=\cap_{j=i}^{\infty}\left[M_{j}^{i} \cup\left(A \backslash \bar{A}_{i}\right)\right] .
$$

Evidently for every $j=1,2, \ldots$ the set $M_{j}^{i} \cup\left(A \backslash \bar{A}_{i}\right)$ is an open and dense subset of $\mathrm{A}$.

This completes the proofo

With each $P \in A$ we associate the number $i_{P}=\min \left\{i: P \in A_{i}\right\}$.

Lemma 3.5 Let $P \in N_{i}$. Then for every $j \geq i_{P}$ holds $P \in M_{j}$.

Proof: By $P \in N_{i}$ we get $i \geq i_{P}$ and $P \in M_{j}$ whenever $j \geq i$.

Let $i_{P} \leq j \leq i$. Then $P \in A_{j}$ and $P \notin\left(A_{i} \backslash \bar{A}_{j}\right)$. If we suppose that $P \notin M_{j}$ then $P \notin M_{j} \cup\left(A_{i} \backslash \bar{A}_{j}\right)$, whereby $P \notin N_{i}$.

The lemma is provedo

We are ready to prove one of the main theorems in the paper

Theorem 3.6 There ezists a dense and $G_{\delta}$ subset $M$ of the set $A$ such that for every $P \in M$ holds that the mapping $F$ is Kuratowsi continuous at $P$. 
Proof: Let us take an arbitrary point $P \in N_{i}$ for some i and a sequence $\left\{P_{n}\right\}_{n \geq 1}$ such that $P=\lim _{n \rightarrow \infty} P_{n}$. We are going to prove that $F(P)=$ $\lim _{n \rightarrow \infty} F\left(P_{n}\right)$ in Kuratowski sense.

1. Let $x \in F(P)$.

We can find $\mathrm{j}$ such that $x \in$ int $B_{j}$. Then there exists a nghb $W$ such that $x \in W \subset B_{j}$. By lemma 3.5 we have that $P \in M_{j}$. Following theorem 2.11 we get that the restricted mapping $F_{j}$ is lower semi- continuous at the point P. From the theorem 3.3 and definitions 3.1 and 3.2 we a derive a sequence $\left\{x_{n}\right\}_{n \geq 1}$ such that for enough large $\mathrm{m} x_{m} \in F_{j}\left(P_{m}\right)$ and $x=\lim _{n \rightarrow \infty} x_{n}$. By the last convergence from a fixed number $m_{0}$ on we have $x_{m} \in W \subset B_{j}$. According to a lemma 1.9 in [8] we obtain $x_{m} \in F\left(P_{m}\right)$.

2. Let us take a sequense $\left\{x_{k}\right\}_{k \geq 1}$ such that $x_{k} \in F\left(P_{m_{k}}\right)$, where $m_{1}<$ $m_{2}<\ldots$ and $x=\lim _{k \rightarrow \infty} x_{k}$.

We assume that $x \notin F(P)$. Since $F(P)$ is a closed set we could determine two open sets $\mathrm{V}$ and $\mathrm{W}$ such that $\mathrm{W}$ is bounded, $x \in W, F(P) \subset V$ and $W \cap V=\emptyset . P \in N_{i}$ therefore there exists $j \geq i_{P}$ such that $x \in W \subset$ int $B_{j}$. By lemma 3.5 $P \in M_{j}$ and from theorem 2.11 we get that the restricted mapping $F_{j}$ is upper semi-continuous at the point $\mathrm{P}$. This means that there exists a number $k_{1}$ such that for all $k \geq k_{1}$ holds $F_{j}\left(P_{m_{k}}\right) \subset V$. Also there is a number $k_{2}$ such that $x_{k} \in W$, whenever $k \geq k_{2}$. Let $k \geq \max \left\{k_{1}, k_{2}\right\}$, again taking into account lemma 1.9 from [8] we get that $x_{k} \in F_{j}\left(P_{m_{k}}\right) \subset V$, which is a contradiction, i.e. $x \in F(P)$.

Having in mind the facts 1 . and 2. also a definition 3.2 and this that the sequence $\left\{P_{n}\right\}_{n \geq 1}$ was chosen arbitrary we obtain that the mapping $\mathrm{F}$ is Kuratowski continuous at the point $P$.

Up to now we have that if $P \in N_{i}$ for some i then $\mathrm{F}$ is Kuratowski continuous at this point. So the points where we have not eventually Kuratowski continuity belong to the set

$$
\cup_{i=1}^{\infty}\left(A_{i} \backslash N_{i}\right)
$$

which is of the first Baire category.

The theorem is proved $\diamond$

Now we shall deal with the same question of Kuratowski contonuity but for the weakly efficient mapping FW. Because of that we do not need anymore of the restriction $l \geq N$ but only $|T| \geq N$. We shall state several definitions. 
Let us consider the following metric on the set int $L_{Z}$ :

$$
\begin{aligned}
& r(x, y)=\|x-y\|+|1 / f(x)-1 / f(y)|, \text { where } \\
& f(x)=\inf \left\{\|x-y\|, y \in \Theta \backslash \operatorname{int} L_{Z}\right\}
\end{aligned}
$$

This is a metric which turns int $L_{Z}$ into a complete metric space and generate in it the same topology as the original norm in $\Theta$. We shall restrict our considerations over the set int $L_{Z}$ with the metric $r(x, y)$.

We define the set $A W=\left\{\sigma \in\right.$ int $\left.L_{Z}: F W(\sigma) \neq \emptyset\right\}$. Obviously

$$
A W=\cup_{i=1}^{\infty} A W_{i} \text {. }
$$

In [7] we have proved that $A W_{i}$ are closed subsets of int $L_{Z}$ and $A W_{i} \subset$ $A W_{i+1}, i=1,2, \ldots$. Also in [10], we have got that the set int $A W \neq \emptyset$ is dense in $L_{W}$. We would like to remind that if the set $\mathrm{A}$ is a closed subset of a topological space $\mathrm{X}$, then the set $A \backslash \operatorname{int} A$ is nowhere dense in X.

Proposition 3.7 The set $\cup_{i=1}^{\infty}$ int $A W_{i}$ is a nonempty and dense subset of the set int $A W$.

Proof: If for each $i=1,2, \ldots$ int $A W_{i}=\emptyset$ having in mind that $A W_{i}$ are closed we shall obtain that the set AW is of the first Baire category. But int $A W \neq \emptyset$ so there exists a number $i_{0}$ such that for all $i \geq i_{0}$ holds int $A W_{i} \neq \emptyset$.

Let us assume that there exists a nghb $\mathrm{V}$ with the property

$$
V \subset \operatorname{int} A W \backslash \cup_{i=1}^{\infty} \operatorname{int} A W_{i}
$$

and take any point $\sigma \in V$. If $\sigma \in A W_{i}$ for some $\mathrm{i}$, by the assumption we get that $\sigma \in A W_{i} \backslash \operatorname{int} A W_{i}$, whereby $V \subset \cup_{i=1}^{\infty} A W_{i} \backslash \operatorname{int} A W_{i}$, or the nghb $\mathrm{V}$ is a subset of a first Baire category set, which is a contradiction, i.e. the set $\cup_{i=1}^{\infty} i n t A W_{i}$ is a dense subset of the set int $A W$.

The proof is completed $\diamond$

Now taking into account that for each $\sigma \in \Theta$ the set $F(\sigma)$ is closed, making the same construction of new $G_{\delta}$ subsets with $\sigma$ insted of $\mathrm{P}$ and repeating the whole proofs of lemma 3.5 and theorem 3.6 with the sets $\cup_{i=1}^{\infty}$ int $A W_{i}$, int $A W_{i}$ and $M W_{i}, i=1,2, \ldots$ insted of $\mathrm{A}, A_{i}$ and $M_{i}, i=1,2, \ldots$ in the same way we get directly the next basic theorem in our paper.

Theorem 3.8 There exists a dense and $G_{\delta}$ subset $M W$ of the set $L_{W}$ such that for every $\sigma \in M W$ holds that the mapping $F W$ is Kuratowski continuous at the point $\sigma$. 
In this case we have not any restrictions on the vector optimization problems, which is due to the closedness of the set of weakly efficient points. According to theorem 2.13 in a dense subset of the set $L_{M}$ the sets of efficient points are closed and the question of Kuratowski continuity in these problems is still open. Looking at the statements in this paragraph we found that the Kuratowski convergence in the image space was the tool which gave us the possibility to prove generic well-posedness of the problems $L V M(\sigma)$ and $L V W(\sigma)$.

\section{References}

[1] Sawaragi Y., Nakayama H., Tanino T. Theory of multiobjective optimization. Math.in Sci. and Eng., Vol. 176, Acad. Press Inc., 1985.

[2] Bednarczuk E. Some stability results for vector optimization problems in partially ordered topological vector spaces. to appear in Proceedings of the first worold congress of nonlinear analysis, 1992.

[3] Brosowski B. Parametric semi-infinite optimization. Frankfurt am Main, Bern, Lang, 1982.

[4] Bednarczuk E. Well posedness of vector optimization problems. preprint.

[5] Tanino T., Sawaragi Y. Stability of nondominated solutions in multicriteria decision making. JOTA 30(2), 1980, 229-253.

[6] Lucchetti R. Well posedness, towards vector optimization. preprint.

[7] Todorov M. Linear vector optimization. Properties of the efficient sets. Serdica-Bulgaricae mathematicae publicationes. 18 (1992) 179-185.

[8] Todorov M. Well-posedness in the linear vector semi-infinite optimization. Proceedings of the tenth international conference on multiple criteria decision making. Taiwan, Taipei, July 19-24, 1992, (Volume IV), p. $1-10$.

[9] Attouch H., Wets R. Quantative stability of variational systems: I. The epigraphical distance. WP-88-8, IIASA, February 1988. 
[10] Todorov M. Existence of the solutions of the linear vector semi-infinite optimization problems. Mathematica Balkanica, New series Vol. 4, 1990, Fasc. 4, p. 390-395.

[11] Fort M.K. Points of continuity of semi-continuous functions. Publ. Math. Debrecen,2, 1951, 100-102.

[12] Christov G., Todorov M. Semi-infinite optimization. Existence and uniqueness of the solutions. Math. Balkanica, New Series, 1988, Fask $2-3$.

[13] Wegmann K. Some properties of the peak-set-mapping. Journal of Approximation theory 8, 262-284, 1973.

[14] Kuratowski K. Topologie I, II. Warszawa, 1961. 